\title{
Strength of visual interpolation depends on the ratio of physically specified to total edge length
}

\author{
THOMAS F. SHIPLEY \\ University of Georgia, Athens, Georgia \\ and \\ PHILIP J. KELLMAN \\ Swarthmore College, Swarthmore, Pennsylvania
}

\begin{abstract}
We report four experiments in which the strength of edge interpolation in illusory figure displays was tested. In Experiment 1, we investigated the relative contributions of the lengths of luminance-specified edges and the gaps between them to perceived boundary clarity as measured by using a magnitude estimation procedure. The contributions of these variables were found to be best characterized by a ratio of the length of luminance-specified contour to the length of the entire edge (specified plus interpolated edge). Experiment 2 showed that this ratio predicts boundary clarity for a wide range of ratio values and display sizes. There was no evidence that illusory figure boundaries are clearer in displays with small gaps than they are in displays with larger gaps and equivalent ratios. In Experiment 3, using a more sensitive pairwise comparison paradigm, we again found no such effect. Implications for boundary interpolation in general, including perception of partially occluded objects, are discussed. The dependence of interpolation on the ratio of physically specified edges to total edge length has the desirable ecological consequence that unit formation will not change with variations in viewing distance.
\end{abstract}

In a world of discrete surfaces and objects, the distance between points will necessarily be related to the likelihood that those points are part of the same surface or object. Such a relationship would also hold for points in the projections of surfaces and objects. However, when spatial gaps are projected to observers, the projected distance between two points depends on viewing distance. Processes determining the unity of separate projected areas, such as edge interpolation in occlusion and illusory figure cases (Kellman \& Shipley, 1991), might thus be expected to involve some sort of perceptual constancy.

Alternatively, perception of unity across spatial gaps might depend on proximal variables, such as projected separation, which change with viewing distance. This possibility would have the unfortunate consequence that the unity or disunity of projected areas might change with viewing distance.

An interesting possible basis for constancy was suggested in one unit-formation ${ }^{1}$ context by Gillam (1981). In her experiments, subjects were shown two separated displays that were parallel projections of objects rotating in depth. Under parallel projection, the axis of rotation is specified; however, the direction of rotation is unspeci-

This research was supported by NSF Research Grants BNS 85-19851 to P.J.K. and BNS 89-13707 to P.J.K. and T.F.S, a James McKeen Cattell sabbatical award, and a Swarthmore College Faculty Research Grant to P.J.K. Portions of this research were presented at the 29th Annual Meeting of the Psychonomic Society (1988). We wish to thank Julia Wong and Jessica Hines for help in collecting data. Reprint requests should be addressed to Thomas F. Shipley, Department of Psychology, University of Georgia, Athens, GA 30602. fied. At any given time, it is possible to see the two objects as rotating either in the same direction or in opposite directions. Early studies showed a strong propensity for certain displays to rotate in the same direction (Gillam, 1972). Most relevant to the present research, studies of spatial separation suggested that the size and spacing of parts trade off in determining whether or not two parts are grouped. The tradeoff appears to occur in such a way that grouping remains constant across changing viewing distances. Gillam found that that the perceived unity of the two rotating lines was a function of the ratio of the length of the lines to the distance between them. The amount of time the two lines appeared to be rotating in the same direction remained constant across displays with similar ratios of edge length to edge separation.

Little is known about the relationship between spatial variables and perceived unity in static unit formation. Kellman and Shipley (1991) have proposed that a number of unit-formation phenomena, including partially $\alpha$ cluded figures and illusory figures, derive from a single process. They conjectured that some distance or proximity metric affects unit formation, but they did not give details. Some evidence suggests that projective size affects boundary perception (Dumais \& Bradley, 1976; Siegel \& Petry, 1991), with smaller retinal sizes giving clearer illusory edges; however, clarity may decrease with very small (less than $1^{\circ}$ of arc) figures (Siegel \& Petry, 1991). If Gillam's notion applies to boundary interpolation, on the other hand, then some ratio of edge length to edge separation may best predict perceived unity. In the present investigation, we attempted to address these questions by 
using illusory figures. Magnitude estimations of edge clarity were assessed in illusory contour displays. Such magnitude estimation procedures have been used effectively in prior research (Day \& Kasperczyk, 1983; Dumais \& Bradley, 1976; Shipley \& Kellman, 1990). In our series of studies, we attempted to assess the role of size and separation of the physically given edges that give rise to illusory contours and figures.

Illusory figures were chosen as a natural vehicle for testing hypotheses about effects of spatial separation. First, there is strong evidence that illusory figures are produced by a unit-formation process that is also responsible for a variety of phenomena including the perception of partly occluded objects (Kellman \& Shipley, 1991; Shipley \& Kellman, in press). Thus, studying the effects of spatial separation in illusory figures is likely to produce results that apply generally to a large class of phenomena. Second, illusory edges are a robust perceptual phenomenon whose quantitative variation can be assessed readily (Dumais \& Bradley, 1976).

Prior research has not clearly resolved the effect of spatial separation of inducing edges on the perception of illusory figures. Two spatial variables may be related to the perceived clarity of the illusory figures: Inducing element size (length of luminance-defined edge; in illusory figures whose corners are specified by circular inducing elements, e.g., Figure 1, the luminance-defined edge would be the radius of the inducing element) and inducing element spacing (the gap between luminance-defined edges). It would hardly be surprising to find that inducing element separation affected perceived clarity. From a theoretical viewpoint, models of illusory figure perception based on interpolation of edges between inducing elements (Grossberg \& Mingolla, 1985; Kellman \& Shipley, 1991) place some limits on the range over which the visual system can interpolate edges. Empirically, it is obvious that inducing elements can be moved far enough apart so that no illusory edge will be seen between them. It is less clear what role the size of inducing elements may play and how their effects may be related to those of separation. For example, do the size and spacing of the inducing elements exert independent effects, or do they interact somehow? One possibility already noted is that the ratio of specified to total edge length determines edge clarity.

Only a few studies have systematically explored the variables of size and spacing in illusory figure displays. Petry, Harbeck, Conway, and Levey (1983) asked subjects to rate the clarity of illusory figures in a set of displays in which the number and size of inducing elements was varied. Increasing the number of inducing elements resulted in an increase in the illusory figure clarity ratings. They also found that increasing the size of the inducing element (which increased the length of the luminancespecified contour around the illusory figure) produced higher clarity ratings. All of the illusory figures Petry et al. (1983) used were the same size. As a result, it is difficult to determine the effect of size or number of inducing elements independent of the effect of distance between inducing elements. When the number of inducing elements around a figure increases, the average distance between elements necessarily decreases. By the same token, when the size of an inducing element is increased, the distance between elements is necessarily decreased. However, Siegel and Petry (1991) reported an experiment in which they independently varied both the number of inducing elements and the size of the illusory figures. They found that these two variables interact. Sharpness or figural clarity would increase with an increase in the number of inducing elements but decrease with an increase in the size of the illusory figure. Such a finding is consistent with the hypothesis that interpolation is determined by both the size and spacing of inducing elements. Changing the number of inducing elements changes a number of properties of an illusory figure display-distance between inducing elements may not be the only factor that influences interpolation.

The third relevant study addressed the effects of retinal and real size on the perception of illusory figures. Dumais and Bradley (1976) asked subjects to rate the clarity of illusory triangle displays in which both the distal size of the illusory figure and the distance from the subject to the display were varied. The illusory triangles were identical in shape. Distal size differences were achieved by magnifying the basic display. The distal and retinal sizes were varied orthogonally. Subjects rated edge clarity higher in displays of small visual angle $\left(1.2^{\circ}\right)$ than they did in displays of large visual angle $\left(18.9^{\circ}\right)$. Displays of intermediate size were given ratings that fell along this continuum. They found no systematic effect of distal size, independent of viewing distance, suggesting that any effect of inducing element separation was due to retinal separation. Other researchers had previously noted an increase in clarity with decreasing retinal size (see Coren, 1972, for a review), but Dumais and Bradley's (1976) experiment provided more systematic evidence for the effect. The study does not allow us to determine the independent effects of size and spacing of inducing elements because these two factors were not varied independently; displays with large distances between inducing elements contained large inducing elements. However, the results suggest that size and spacing do not trade off in such a way that clarity remains constant across different viewing distances.

\section{EXPERIMENT 1}

Neither the experiments of Petry (Petry et al., 1983; Siegel \& Petry, 1991), nor of Dumais and Bradley (1976), allow us to assess the relative contributions to illusory figure clarity of size of inducing element (length of physically specified contour) and distance between inducing elements (length of interpolated contour). In these studies, changes across displays in one factor were correlated with changes in the other factor or some other factor such as number of elements. In Experiment 1, we investigated the effects of each factor by using a set of illusory figure displays in which the size and spacing of inducing elements were independently varied. 


\section{Method}

Subjects. Twenty-four undergraduates at the University of Pennsylvania served as subjects in 30 -min individual testing sessions. Each subject received $\$ 2.50$ for participating.

Apparatus. All stimuli were designed and presented using a Commodore Amiga computer with a Commodore $1080(20 \mathrm{~cm}$ high $\times$ $25 \mathrm{~cm}$ wide) RGB monitor. When the computer's high resolution mode was used, the screen resolution was 400 vertical $\times 640$ horizontal pixels ( 25 pixels $/ \mathrm{cm}$ ). All stimuli were presented to each subject in a random order, with the constraint that for any given subject who received a particular random order, there was another subject who was presented with the stimuli in the reverse order.

The subjects were positioned $150 \mathrm{~cm}$ from the monitor. The only source of light in the room, other than the monitor, was a screened 100-watt bulb positioned above and behind the monitor. This lighting arrangement reduced reflections from the monitor.

Stimuli. Each display consisted of four white three-fourths-circle inducing elements (luminance: $30.4 \mathrm{fL}$ ) arranged on a black background (luminance: $0.0 \mathrm{fL}$ ) to form a potential illusory square. Four separations between inducing elements were used: $1,2,3$, and $4 \mathrm{~cm}$ $\left(0.38^{\circ}, 0.76^{\circ}, 1.14^{\circ}\right.$, and $1.52^{\circ}$ of arc $)$. Three different-sized inducing elements were used, with radii of $0.8,1.4$, and $2 \mathrm{~cm}\left(0.30^{\circ}\right.$, $0.57^{\circ}$, and $0.76^{\circ}$ of arc). The four separations were factorially combined with the three inducing elements' sizes to yield a total of 12 displays. Figure 1 shows the displays used for Experiment 1. The leftmost column shows the three different inducing elements' sizes separated by the largest separation; the separation decreases in successive columns from left to right.

Procedure. The instructions and rating scale used in this experiment were identical to those used in previous work that required the assessment of contour clarity (Shipley \& Kellman, 1990).

The subjects were seated in front of the monitor and given the following instructions:

This is an experiment involving illusory figures. I will show you some displays that contain illusory figures and some displays that do not contain illusory figures. [The subject was then shown a standard Kanizsa triangle display (the length of the illusory triangle side and element triangle was $2.9 \mathrm{~cm}$ or $1.11^{\circ}$ of arc); the radius of the circular inducing elements was $0.7 \mathrm{~cm}$ or $0.27^{\circ}$ of arc); the experimenter pointed to the display and indicated the outer boundaries of the illusory triangle.] This is an example of an illusory figure; the central triangle is the illusory figure. It is important to note two of its properties. First, you can see the entire triangle, and second, you can see a clear edge even in the areas between the white areas (the inducing elements) in the display. [The experimenter then showed a display consisting of multiple white dots placed at the vertices of a triangle similar in size and location to the illusory triangle they had just seen. Such displays do not produce illusory figures. The experimenter then pointed to the area between the white dots.] This is not an illusory figure. While you could imagine a triangle formed by the three sets of dots, most people do not see a complete figure with clear edges between the white areas. Because the display does not contain a triangle with clear edges, it does not contain any illusory figure.

Similar instructions were given with an illusory figure display containing an illusory rectangle [the illusory figure was $4.4 \times 7.0 \mathrm{~cm}$ $\left(1.68^{\circ} \times 2.67^{\circ}\right.$ of $\left.\operatorname{arc}\right)$; the circular inducing elements had a radius of $2.0 \mathrm{~cm}\left(0.76^{\circ}\right.$ of arc) $]$ and a similar display in which only the outlines of the inducing elements were shown. (Such outline inducing element displays do not produce illusory figures). The subject was then asked if he/she understood what was meant by "illusory figure." If the subject answered "no,"' the experimenter showed the displays a second time. The experimenter then explained the subject's task as follows:

You will now see several displays similar to the displays that you have just seen. We would like you to indicate whether or not you see an illusory figure in the display. If you do see an illusory figure, we would like you to rate the clarity of the figure using a scale from one to ten. If the figure is as clear as the first figure that you saw, you should rate it a ten; if it is less clear, you should give it a lower number. Do you have any questions?

Each display was presented for at least $10 \mathrm{sec}$ and was removed only after the subject had responded. A 5-sec interval, during which the screen was blank, separated the experimental displays. Before the experimental displays were presented, 10 practice displays were presented that allowed the subject to become comfortable rating il-

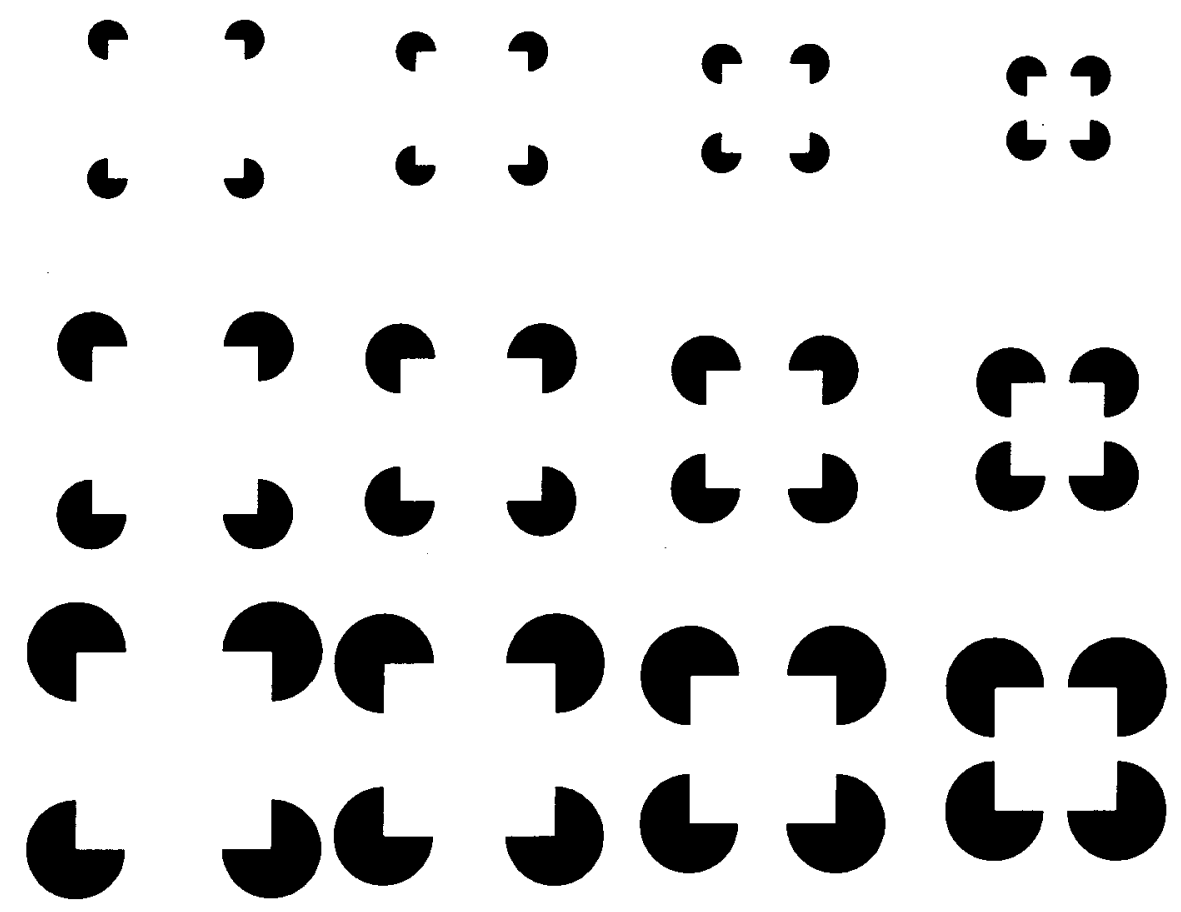

Figure 1. The 12 displays used in Experiment 1. 
lusory figure displays and ask any questions. We included two standard illusory figure displays in the set of practice displays. One was an illusory circle formed from radiating lines, a figure that other investigators have found to produce clear illusory figures (Petry et al., 1983); the other standard was an illusory square formed by placing crosses at each corner, a figure that produces weak or no illusory figures (Day \& Kasperczyk, 1983). The subjects' responses to these figures were to be used to screen out subjects who misunderstood the task or had unusual response tendencies. The criteria were that the radiating-lines display should be rated no lower than 6 and the cross display no higher than 2 .

\section{Results}

No subjects were excluded from the analysis for failing to meet the criteria discussed in the procedure section.

For the purposes of analysis, responses of "no illusory figure" were assigned a rating of 0 . The mean ratings for each figure are shown in Figure 2. The subjects' ratings were subjected to a two-way analysis of variance (ANOVA) with repeated measures, with element size and spacing as factors. The results of the analysis showed that illusory figure ratings increased as the size of the inducing elements increased $[F(2,38)=80.2, p<.0001]$ and as the distance between these elements decreased $[F(3,57)=53.6, p<.0001]$. The interaction between these two factors was also significant $[F(6,114)=2.36$, $p<.05$ ].

Planned comparisons revealed significant differences between each of the successive element sizes and element separations.

The interaction between inducing element size and spacing suggests that illusory figure clarity is not simply an additive function of the two features. An illusory figure's perceived clarity cannot be determined solely on the basis of the inducing elements' size or separation. What accounts for the interaction between the inducing element size and spacing? One possibility is that the size and spacing trade off, as suggested by Gillam (1981) in a different unit-formation context. If so, perceived clarity would



Figure 2. Mean clarity rating for each size inducing element plotted as a function of inducing elements separation. The vertical bars represent $1 S E(n=24)$. (From Kellman \& Shipley, 1991, Figure 31. Copyright 1990 by the Academic Press. Reprinted by permission.) be constant across displays with equivalent ratios of size to spacing.

To test this hypothesis, size and separation were combined to form a third variable. This variable $(V)$ was the ratio of two times the radius of the inducing element $(2 r)$ to the distance between inducing element $(d)$ plus two times the radius of the inducing element $(2 r)$. Put more simply, $V$ is the fraction of the whole edge that is physically specified: $V=2 r /(d+2 r)$. It is also the fraction of the illusory figure's edge that does not need to be interpolated by the visual system.

To determine whether the subjects' responses were determined by this ratio, a one-way ANOVA was run on ratings of all 12 stimuli. The result was a main effect of stimulus type $[F(11)=17.2, p<.0001]$, in which $95 \%$ of the variance attributable to stimulus type was consistent with a linear trend.

Figure 3 shows the mean clarity rating as a function of the ratio variable. The graph makes clear two important aspects of the data. The first is that the mean ratings now lie along a straight line. The best-fitting linear regression line had an intercept of -1.53 with a slope of 13.46 $(r=.98)$. The second is that the clarity ratings of displays of approximately equivalent ratios did not differ, even though the displays differed in both inducing element size and distance.

\section{Discussion}

The size and spacing of inducing elements both seem to affect the perceived clarity of illusory figures. The clarity of an illusory figure increases with increasing inducing element size when spacing is constant, and clarity decreases as the distance between inducing elements increases when size of inducing elements is constant. But the two variables do not appear to have independent effects. The effect of each variable is best expressed in terms of a higher order variable. Illusory figure clarity appears to be predicted by a ratio of the specified edge of the illusory figure to the overall length of the edge of the illusory figure. As the proportion of an illusory figure that must be interpolated decreases, the perceived clarity of the interpolated edge increases.

The dependence of perceived edge clarity on such a ratio implies that whether and how well illusory figures form will not in general be changed by changes in viewing distance. This outcome parallels that found by Gillam (1981) in experiments on the perceived coherence of separate rotating figures.

The relationship between the ratio of specified to total edge length and illusory figure strength appears to be linear. That illusory figure clarity is a function of the ratio of specified to total edge length is consistent with Petry's results (Petry et al., 1983; Siegel \& Petry, 1991), in which an increase in either number or size of inducing elements resulted in an increase in illusory figure clarity. An increase in either the number or size of inducing elements will result in an increase in the specified contour in the display, with a resulting increase in the ratio. 




Figure 3. Mean clarity ratings from Experiment 1 plotted as a function of the ratio of specified to total illusory figure edge length $(n=24)$. (From Kellman \& Shipley, 1991, Figure 32. Copyright 1990 by the Academic Press. Reprinted by permission.)

\section{EXPERIMENT 2}

The results of Experiment 1 are consistent with both the hypothesis that interpolation strength is a function of the ratio of specified to unspecified contours and with the findings of Petry et al. (1983) and Siegel and Petry (1991). However, they are not consistent with Dumais and Bradley's results. Dumais and Bradley (1976) found a decrease in the perceived clarity of an illusory figure with an increase in retinal size, but we found no effect of absolute figure size: Our subjects rated displays with the same ratio but differing size as equivalent. The failure of Experiment 1 to replicate Dumais and Bradley (1976) is puzzling. It was not, however, explicitly designed to test the relationship between the ratio of specified contour and perceived clarity of illusory figures. Thus, there were only a few displays with roughly equivalent ratios, and these came from a rather narrow range of size and ratio. Experiment $2 \mathrm{~A}$ tested the relationship between ratio and figure size over a broader range of ratios.

One possible explanation for the discrepancy between our findings and Dumais and Bradley's (1976) is that a limited range of display sizes were used in Experiment 1. Dumais and Bradley used displays that ranged from $1.2^{\circ}$ of arc to $18.9^{\circ}$ of arc. Within the range of displays that we used, their subjects did rate small displays higher than the larger ones, but the differences were not very large. Experiment 2B, by using a larger range of illusory figure sizes, tested the possibility that our failure to obtain a size effect was due to the limited range of sizes.

\section{Experiment 2A}

\section{Method}

Subjects. Twenty undergraduates at the University of Pennsylvania served as subjects in 30-min individual testing sessions. Each subject received $\$ 2.50$ for participating.
Apparatus. The apparatus used for Experiment 2A was the same as the one described in Experiment 1.

Stimuli. Similar to the stimuli used in Experiment 1, each display consisted of four three-fourths-circle inducing elements arranged so that an illusory square could be seen. Three different illusory square sizes were used: 2,4 , and $8 \mathrm{~cm}\left(0.76^{\circ}, 1.52^{\circ}\right.$, and $3.05^{\circ}$ of arc). For each illusory square size, nine illusory figure displays, with ratios from .1 to .9 in equal intervals, were constructed. The inducing elements necessary for these displays ranged in size from a radius of $3.6 \mathrm{~cm}\left(1.37^{\circ}\right.$ of arc) for an illusory square of $8 \mathrm{~cm}$ and a ratio of .9 , to a radius of $0.1 \mathrm{~cm}(2.3 \mathrm{~min}$ of arc) for an illusory square of $2 \mathrm{~cm}$ and a ratio of .1 . Nine of the displays are shown in Figure 4. The different sized illusory figures appear in each row. The displays in each column have ratios of $.9, .5$, and .1 , from left to right.

Procedure. The procedure and instructions used in Experiment $2 \mathrm{~A}$ were the same as in Experiment 1. Each subject viewed all 27 displays.

\section{Results}

No subjects were excluded from the analysis for failing to meet the criterion discussed in the procedure section.

As in Experiment 1, responses of "no illusory figure" were assigned a rating of 0 . The mean ratings for each illusory square size and ratio are shown in Figure 5. The subjects' ratings were subjected to a two-way ANOVA, with ratio and size of illusory square as factors. The results of the analysis showed that illusory figure ratings increased as the ratio increased $[F(8,152)=202.0, p<$ $.0001]$ and as the size of the illusory square increased $[F(2,38)=5.28, p<.01]$. The interaction between these two factors was not significant $[F(16,304)=1.38$, $.10<p<.15$ ].

The clarity of the illusory square decreased as its ratio decreased. With the exception of the highest ratios, in which the difference in clarity between a ratio of .9 and .8 was only marginally significant $[F(1,152)=2.76, p=$ .09 ], there was a significant increase in clarity with each increase in ratio [all $F \mathrm{~s}(1,152) \geq 7.41, p<.01$ ].

The subjects rated large illusory figures slightly higher than they rated small illusory figures. As can be seen in Figure 5, these differences are most pronounced at the lower ratios. In retrospect, this outcome is not entirely surprising; the inducing elements in the displays with low ratios and small illusory figures were very small. The smallest inducing elements were only a few pixels in radius; the decrease in clarity for the smaller displays may be in part due to a decrease in the display resolution relative to figure size for these displays.

Again, the function relating ratio to perceived clarity appears to be linear; the percentages of variance attributable to a linear trend were $97.7,98.1$, and 95.2 for the 2,4 , and $8 \mathrm{~cm}$ illusory squares. The intercepts for the three functions were $-.86,-.09$, and .41 , and the slopes were $12.87,11.64$, and 11.48 .

\section{Method}

\section{Experiment 2B}

Subjects. Twenty undergraduates at Swarthmore College served as subjects in 30-min individual testing sessions. Each subject received $\$ 2.50$ for participating. 


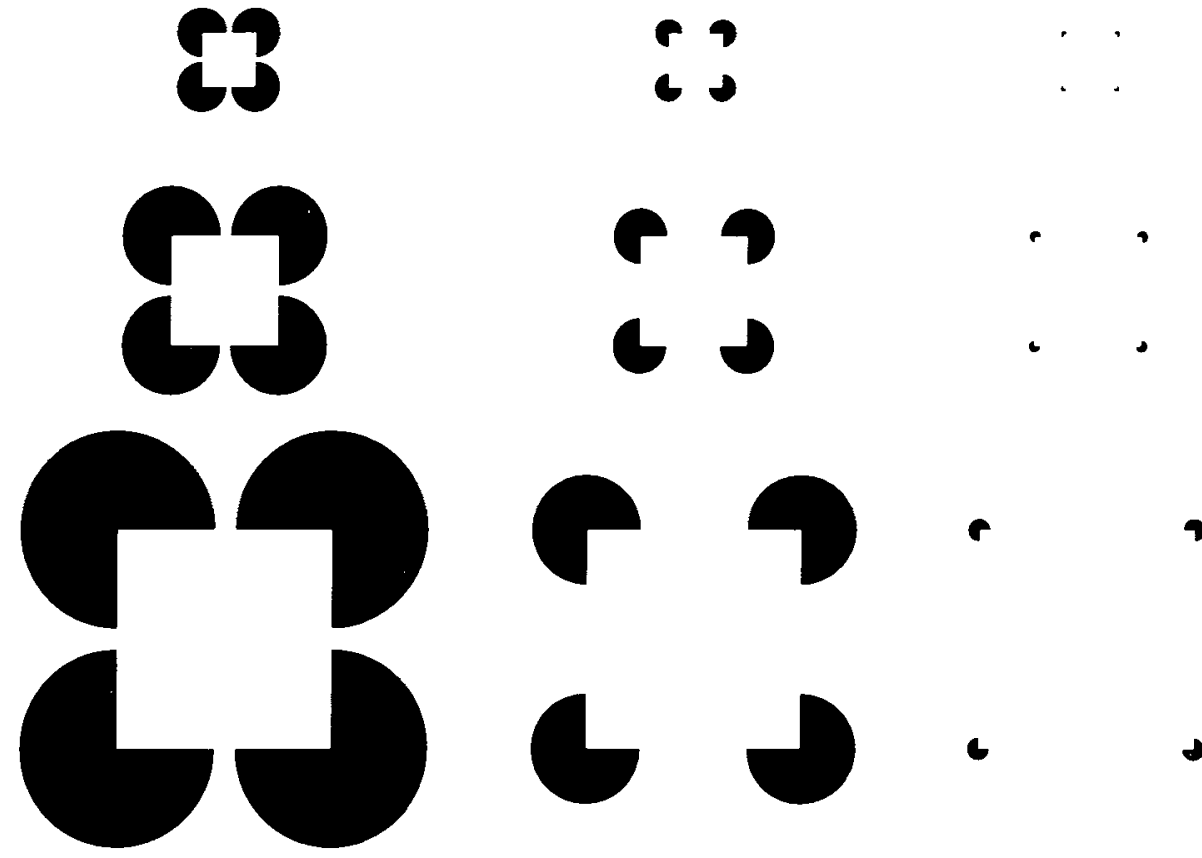

Figure 4. Nine of the 27 displays used in Experiment 2A.

Apparatus. All stimuli were designed and presented using a Macintosh II computer with an E-Machine's TX16 $(25 \mathrm{~cm}$ high $\times$ $33 \mathrm{~cm}$ wide) RGB monitor. This monitor provided a larger display and greater resolution than did the Amiga monitor. The higher resolution allowed us to position the subjects closer to the monitor, further increasing the retinal size of the displays. The screen resolution was $34.25 \mathrm{dots} / \mathrm{cm}$ (808 vertical $\times 1024$ horizontal pixels). All stimuli were presented to each subject in a random order (except where noted).

The subjects were positioned $50 \mathrm{~cm}$ from the monitor. The room was dark except for the illumination provided by the monitor.

Stimuli. A set of 12 illusory square displays, similar to those used in Experiments 1 and $2 \mathrm{~A}$, were constructed using four different illusory square sizes and three different ratios. The ratios used were $.3, .5$, and .7 . The illusory square edge lengths were 11.7 , $5.8,2.9$, and $1.4 \mathrm{~cm}$. With a viewing distance of $50 \mathrm{~cm}$, the angular sizes of the illusory figures were $13.4^{\circ}, 6.65^{\circ}, 3.32^{\circ}$, and $1.60^{\circ}$ of arc. The inducing elements were white figures (luminance: $27.6 \mathrm{fL}$ ) on a black background (luminance: $0.0 \mathrm{fL}$ ).

Procedure. The procedure and instructions used in Experiment $2 \mathrm{~B}$ were the same as in Experiment 1, with the following exceptions. The subjects were instructed to give a display a rating of 0 if they saw no illusory figure and use the scale from 1 to 10 to rate any figure that was seen. Each subject viewed the set of displays twice. Displays were presented using a blocked design so that all 12 displays were presented in random order before any one display was presented a second time.

\section{Results}

Figure 6 shows the mean ratings for each ratio as a function of illusory figure size. Once again, the clarity of an illusory figure appears to track the ratio of specified to total edge length. The subjects' ratings were subjected to a three-way ANOVA, with ratio, size of illusory square, and repetition as factors. The results of the analysis showed that illusory figure ratings increased as the ratio increased $[F(2,38)=78.54, p<.0001]$ and as the size of the illusory square increased $[F(3,57)=5.08, p<$ $.005]$. There was also a main effect of repetition $[F(1,19)=6.33, p<.05]$.

As in Experiments 1 and 2A, clarity appears to be a linear function of ratio. The percentage variance attributable to a linear trend for the four illusory square sizes $(1.4,2.9,5.8$, and $11.7 \mathrm{~cm})$ was above 98 . The intercepts for the four functions were $1.60,0.64,1.31$, and 0.43 , and the slopes were $15.37,12.00,10.82$, and 12.55 .

Displays with high ratios were rated considerably higher than displays with lower ratios. Collapsing over figure size, the mean rating for displays with a ratio of .7 was 8.91. Mean ratings for displays of ratios .5 and .3 were

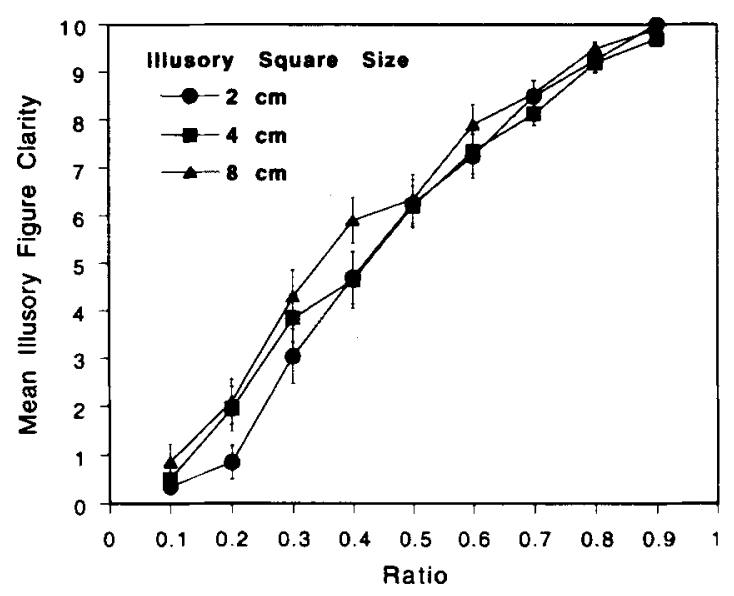

Figure 5. Mean clarity ratings from Experiment $2 A$ plotted as a function of the ratio of specified to total illusory figure edge length. The vertical bars represent $1 S E(n=20)$. 




Figure 5. Mean clarity ratings from Experiment $2 \mathrm{~A}$ plotted as a function of the ratio of specified to total illusory figure edge length. The vertical bars represent $1 S E(n=20)$.

6.83 and 3.85, respectively. Each of these differed significantly from the others [all $F \mathrm{~s}(1,38) \geq 26.27, p<$ $.0001]$.

As in Experiment 2A, the smallest displays tended to be rated slightly lower than the other displays. The smallest illusory figures had a mean rating of 6.08 , across ratios, whereas the $2.9-, 5.8-$, and $11.7-\mathrm{cm}$ illusory figures were rated $6.64,6.71$, and 6.70 , respectively.

The interaction between figure size and ratio did not reach significance in Experiment $2 \mathrm{~A}$ but did in Experiment $2 \mathrm{~B}[F(6,114)=3.89, p<.005]$. The pattern of responses to the different-size figures across ratios is very similar to the pattern seen in Experiment $2 \mathrm{~A}$. The clarity difference between small and large displays appears most clearly in the lowest ratio displays. Planned comparisons of the mean ratings for each size within each ratio confirmed this; the only significant differences were between the smallest and the three larger displays for ratio $=.3$ [for these three comparisons, all $F \mathrm{~s}(1,114) \geq 20.33, p<$ .0001 ; all other $F \mathrm{~s}(1,114) \leq 2.10, p>.15]$.

The ratio $\times$ repetition interaction was marginally significant $[F(2,38)=2.52, .05<p<.10]$. The repetition $X$ size and three-way interactions were not significant $(F s<1)$. The main effect of repetition appears to be due to a general decrease in the magnitude of the ratings in the second set of displays. The overall mean for the first block was 6.78, and the mean for the second block was 6.29 . The ratio $x$ repetition interaction appears to be due to a decrease from the first to the second block in the ratings of the displays with a ratio $=.5$.

\section{Discussion}

The pattern of results in Experiments $2 \mathrm{~A}$ and $2 \mathrm{~B}$ are very similar to that of Experiment 1 . Different-size illusory figures with equivalent ratios were judged to be roughly identical in clarity. Although there appears to be a small effect of figure size on the perceived clarity of illusory figures, this effect is quite small relative to the effect of the ratio of specified edge to illusory figure edge. Moreover, the size effect is opposite in direction to the size effect reported by Dumais and Bradley (1976). Over a rather large range of figure sizes, the ratio of a figure appears to be a good predictor of clarity. Figure size, on the other hand, does not appear to be as useful. It is not even clear that the effect of figure size is anything more than a breakdown in the resolution necessary to display small inducing elements on a CRT screen. We will return to the issue of display generation in the general discussion.

\section{EXPERIMENT 3}

The notion that the ratio of specified to specified plus unspecified edge determines illusory contour strength fits the data of Experiments 1 and 2. Our conclusions to this point, however, rest on a single method: magnitude estimation. Because of the possibility of a small size effect (Dumais \& Bradley, 1976), and generally to test for small effects not easily revealed by magnitude estimation, we carried out an additional experiment by using a paired comparison technique.

Experiment 3 tested the robustness of the ratio finding by asking the subjects to judge the relative clarity of two illusory figure displays presented simultaneously. An important advantage of the use of simultaneous pairwise comparisons is that they may be sensitive to minute perceptual differences that are easily lost in a magnitude rating paradigm.

\section{Method}

Subjects. Sixteen undergraduates at Swarthmore College served as subjects in 30-min individual testing sessions. Each subject received $\$ 2.50$ for participating.

Apparatus. The apparatus was the same as in Experiment 2B. The subjects were positioned $120 \mathrm{~cm}$ from the monitor. The room was dark except for the illumination provided by the monitor.

Stimuli. A set of nine illusory square displays, similar to the ones used in Experiments 1 and 2, were constructed using four different illusory square sizes and three different ratios. The ratios used were $.2, .5$, and .8 . The illusory square sizes were $1.46,2.92$, and $5.84 \mathrm{~cm}$. At the viewing distance of $120 \mathrm{~cm}$, the angular sizes of the illusory figures were $0.70^{\circ}, 1.40^{\circ}$, and $2.79^{\circ}$ of arc.

Procedure. The subjects were seated in front of the monitor and read the introduction used in Experiment 1, which explained the difference between seeing and imagining a figure. They were then given the following instructions:

\begin{abstract}
You will now see a number of pairs of displays that may contain illusory figures. If you see illusory figures in both displays, we would like you to indicate which of the two illusory figures appears clearer. If the two displays are precisely identical in clarity, then indicate that. If you only see one illusory figure in the pair of displays, then that one should be indicated as clearer; if you do not see an illusory figure in either display, then report no illusory figure.
\end{abstract}

The subjects were then shown the third and fourth instruction displays from the instruction set used in Experiment 1 (an illusory square display and a similar display with line-drawn inducing elements), as a pair, and asked which of the two appeared clearer. They were allowed to ask questions and were then shown the experimental stimuli.

The subjects were presented with 72 trials in random order. Over the 72 trials, each of the nine illusory figure displays appeared on the left side, with one of the other eight displays on the right. 


\section{Results}

The results were clear and consistent with the findings of Experiments 1 and 2. No subject reported that neither side contained an illusory figure in any display. Table 1 shows the percentage of trials, over all subjects, on which displays at each level of the ratio factor were judged to be clearer than displays at another level. Overall, the subjects judged the display with a greater ratio to be clearer on $83.7 \%$ of the trials. The display with a greater ratio was judged to be less clear on only $8.9 \%$ of the trials. (In the remaining $6.4 \%$ of the trials, the two figures were judged equal.) For all three ratios, the subjects were more likely to judge the greater ratio figure to be clearer (all zs $\geq 10.83, p<.0001$; sign test).

The size of the illusory figure did not have much of an influence on judged clarity. Table 2 shows the percentage of trials in which displays of one size were judged to be clearer than displays of another size. The smaller illusory figure was judged to be clearer on $37.5 \%$ of the trials, whereas the larger was judged to be clearer on $42.9 \%$ of the trials. The subjects were slightly more likely to judge the larger figure to be clearer in trials in which the largest and smallest displays were compared $(z=$ $2.44, p<.02$ ). There was no reliable difference in the other two cases $(z<1)$.

\section{Discussion}

The results of Experiment 3 confirm and extend those of the previous two experiments. Displays with high ratios appeared to have clearer illusory edges than displays with lower ratios. There was no evidence that small illusory figures are clearer than larger displays with equivalent ratios. If anything, there was a small tendency in the reverse direction.

\section{GENERAL DISCUSSION}

In a given illusory figure display, increasing the length of physically given edges increases figure clarity. Likewise, decreasing the separation of inducing edges increases clarity. Describing these two effects separately, however, conceals a deeper generalization. The simplest account of these effects is that clarity depends on the ratio of physically specified edge length to total extent (inducing edge lengths plus extent of separation).

This relationship parallels that found by Gillam (1981) in a different paradigm. When separated figures are presented in rotation under parallel projection, perceived

Table 1

Percentage of All Trials In Which the High Ratio was Judged Clearer Than the Lower Ratio $(H>L)$, the Low Ratio was Judged

Clearer Than the Higher Ratio $(H<L)$, and the High Ratio was Judged Equal to the Lower Ratio $(H=L)$.

\begin{tabular}{|c|c|c|c|c|}
\hline & \multicolumn{3}{|c|}{ Ratio } & \multirow[b]{2}{*}{$M$} \\
\hline & .8 vs. .5 & $.8 \mathrm{vs} . .2$ & .5 vs. .2 & \\
\hline$H>L$ & 77.8 & 90.0 & 83.3 & 83.7 \\
\hline$H<L$ & 15.9 & 6.6 & 4.2 & 8.9 \\
\hline$H=L$ & 6.3 & 3.4 & 12.5 & 7.4 \\
\hline
\end{tabular}

Table 2

Percentage of All Trials in Which the Smaller Illusory Figure was Judged Clearer Than the Large Illusory Figure $(S>L)$, the Large Illusory Figures was Judged Clearer Than the Smaller Illusory Figure $(S<L)$, and the Small Illusory Figure was Judged Equal to the Larger Illusory Figure $(S=L)$ Illusory Figure Sizes (degrees of arc)

\begin{tabular}{lccccc} 
& 0.7 vs. 1.4 & 0.7 vs. 2.8 & 1.4 vs. 2.8 & $M$ \\
\hline$S>L$ & 36.5 & 35.4 & 40.6 & 37.5 \\
$S<L$ & 40.3 & 48.6 & 39.9 & 42.9 \\
$S=L$ & 23.2 & 16.0 & 19.5 & 19.6 \\
\hline
\end{tabular}

unity depends on the ratio of figure size to spacing between figures.

As Gillam (1981) pointed out, such an outcome is ecologically sensible. Although the projected size of elements or the projected size of a gap between elements will depend on the position from which an observer views objects, the ratio of specified to total extent will be invariant across viewing distance. This is a good arrangement for achieving a coherent representation of objects' unity and boundaries, since object unity does not depend on observer position. ${ }^{2}$

The ratio hypothesis accounts for over $95 \%$ of the variance in edge clarity ratings in our experiments. It encompasses illusory figures whose angles range from as small as $0.76^{\circ}$ of arc to as large as $13.4^{\circ}$ of arc, and real sizes from $1.4 \mathrm{~cm}$ to $11.7 \mathrm{~cm}$.

The possibility that retinal size is an independent determinant of edge clarity receives minimal support from these studies. Although small reliable effects were found in Experiments $2 \mathrm{~A}$ and $2 \mathrm{~B}$ for larger visual displays, a more direct assessment using a paired comparison technique (Experiment 3) did not show a clear effect of size. A size effect reported previously (Dumais \& Bradley, 1976) was opposite in direction to the small size effects noted here. One possible explanation for these small and elusive effects involves the means of display generation. In our studies, the small superiority of larger displays was most evident in the smallest displays. This effect may be due to limitations of display resolution that become relatively more consequential at the smallest sizes. On the other hand, Dumais and Bradley (1976) constructed their displays by using paper and ink. Our experience with such displays suggests that minor imperfections in handmade displays become more noticeable in larger versions. This difference might explain why Dumais and Bradley found a reliable superiority of illusory figure clarity for smaller displays.

\section{Implications for Theories of Unit Formation}

Kellman and Shipley (1991; see also Kellman \& Loukides, 1987) put forth an account of boundary interpolation that treats perception of the unity of partly occluded objects and perception of illusory figures in identical fashion. The process they describe works on the local orientations of projected edges, and the apparent differences between illusory figures and partly occluded objects derive from depth assignment of units formed relative to 
other surfaces, not from differences in the unit-formation process. Although the process works for a variety of unitformation phenomena, Kellman and Shipley suggest that the interpolation process exists primarily to deal with partial occlusion, a pervasive problem in ordinary object perception. Gillam's (1981) ecological argument that unit formation should be relatively invariant across viewing position would seem to apply forcefully to the perception of occluded objects. Our data suggest that a ratio principle governs illusory figure perception; in all likelihood, the same ratio principle will apply to occlusion cases. Recent data confirm the equivalence of unit formation in occlusion and illusory figure cases across a wide variety of displays, including randomly generated ones (Shipley \& Kellman, in press).

The present data suggest that models of unit formation should incorporate quantitative variation in interpolation strength based on the ratio of specified to total edge length. Both distance between luminance-defined edges and the length of those edges must be taken into account. Among existing models (Grossberg \& Mingolla, 1985, 1987; Kellman \& Shipley, 1991; Ullman, 1976) only Grossberg and Mingolla's explicitly considers the issue of variations in the strength of the illusory edge. They suggest that an illusory edge results from the cooperative interaction of local-oriented edge units. When two spatially separated units with similar orientation are activated, they, in turn, may activate a bipole unit that causes the activation of intervening units. Grossberg and Mingolla suggest that these bipole units may have different size receptive fields. Different units may be activated by like-oriented edge units over a range of spatial locations. The bipole units with larger spatial ranges require more activation from the likeoriented edge detectors. Grossberg and Mingolla (1987) give the details of the relationship between range and the necessary amount of initial edge unit activity.

Grossberg and Mingolla's $(1985,1987)$ model is a specific instance of a general class of models in which interpolation occurs at several different levels of scale. Multiple levels of scale may also explain the perception of certain illusory figure displays (e.g., Minguzzi, 1987), in which the boundaries of inducing elements are blurry. Interpolation that occurs at the coarsest scale (low spatial frequencies) is sufficient to produce an illusory boundary. The illusory boundary, however, also appears blurry. This paradoxical outcome may be due to the failure of edge interpolation at finer levels of scale (high spatial frequencies). This interpretation implies that the crisp boundaries seen in ordinary illusory figure displays result from edge interpolation at all scales.

Our finding that unit formation seems to be a function of the ratio of specified to unspecified edge length raises a number of interesting issues. First, what specified edge lengths are relevant to the ratio? If illusory contours are a result of local interpolation (Grossberg \& Mingolla, 1985; Kellman \& Shipley, 1991; Ullman, 1976), then specified edges remote from a given site of interpolation may not affect boundary formation. The strength of a

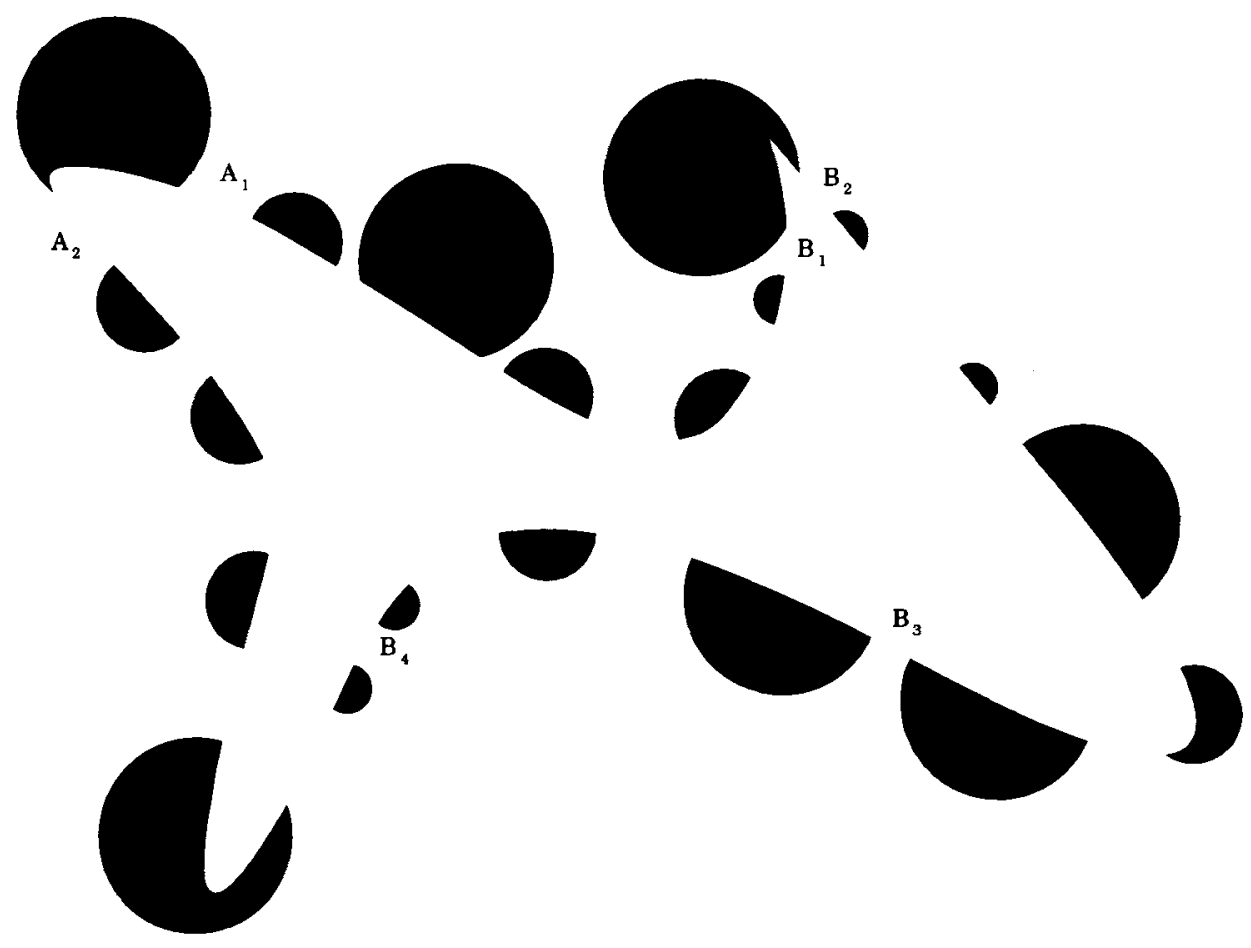

Figure 7. Illusory figure illustrating issues of ratio determination in complex figures. Gaps labeled Al and $A 2$ are equal in size and have the same length of luminance-specified edge on either side (as do gaps B1 and B2), yet the interpolated boundaries do not appear equally clear. The clarity difference may depend on the extent of adjacent luminance-specified edges that are smooth and monotonic (see text). Such edges are greater in $\mathrm{A1}$ and $\mathrm{B1}$ than in $\mathrm{A} 2$ and $\mathrm{B} 2$. 
given boundary segment is determined by its local ratio. Our experimental displays, with equivalent gaps on each side of a regular figure, have equivalent local ratios (i.e., ratios associated with each gap) and global ratios (along the entire figure boundary). In displays with irregular figures and multiple different-sized gaps (as occurs in real world occlusion), determining the ratio for each gap is not straightforward. The relevant length of luminancespecified edge in an illusory figure that is curved or irregular may not simply be the total length of adjacent specified edge. Drawing on our proposal that the interpolated boundary must be monotonic and smooth (Kellman \& Shipley, 1991), perhaps the relevant specified contour is limited to adjacent edges that, combined with the interpolated edge, are smooth and monotonic. Some illustrations are given in Figure 7.

Second, one might ask whether the ratio is the only relevant variable needed to determine the strength of an illusory edge. The ratio does not appear to be sufficient for determining edge strength. This is readily demonstrated by observing the effect of adding thin lines perpendicular to, and terminating at, the illusory edge. Such displays (e.g., the standard Kanizsa triangle) become noticeably weaker when the thin lines are deleted. Such thin lines contribute a very small fraction of the specified edge of the illusory triangle, yet they appear to have a substantial effect on perceived clarity. The number of inducing elements may contribute to the strength of an interpolated boundary independent of the amount of specified contour each element contributes.

How much of the quantitative variation in the strength of interpolation can be explained by the ratio effect? The answer is not yet clear. Other influences purported to affect illusory figure clarity may or may not be separate from ratio effects. These include viewing distance (Coren, 1972), illumination level (Dumais \& Bradley, 1976), and relative orientation of inducing edges (Grossberg \& Mingolla, 1985; Kellman \& Shipley, 1991). The most obvious consequence of varying viewing distance-changing retinal size-seems to have a negligible effect on interpolation strength. An effect of viewing distance could occur because of altered visibility of surface texture. Nakayama, Shimojo, and Silverman (1989) and Rock and Anson (1979) have found that illusory figure formation is much weaker when a textured surface appears at a greater depth than do the illusory figure inducing elements. Perhaps when illusory figure displays are viewed close up, any texture on the surface of the display (e.g., paper fibers in ink-on-paper displays) interferes with boundary formation. Such an explanation may also apply to Dumais and Bradley's (1976) finding that illusory figure clarity increases with decreasing illumination. At low illumination levels, any texture between the inducing elements may not be as visible. The same would be true for any imperfections in hand-drawn displays. Finally, the suggestion that strength of interpolation varies with relative edge orientation (Grossberg \& Mingolla, 1985; Kellman \& Shipley, 1991; Shipley \& Kellman, in press) would seem to be an effect separate from the ratio effect.

\section{REFERENCES}

Coren, S. (1972). Subjective contours and apparent depth. Psychological Review, 79, 359-367.

DAY, R. H., \& KASPERCZYK, R. T. (1983). Amodal completion as a basis for illusory contours. Perception \& Psychophysics, 33, 355-364

Dumais, S. T., \& BRadiey, D. R. (1976). The effect of illumination level and retinal size on the apparent strength of subjective contours. Perception \& Psychophysics, 19, 339-345.

Gillam, B. (1972). Perceived common rotary motion of ambiguous stimuli as a criterion of perceptual grouping. Perception \& Psychophysics, 11, 99-101.

GiLlAM, B. (1981). Separation relative to length determines the organization of two lines into a unit. Journal of Experimental Psychology: Human Perception \& Performance, 7, 884-889.

Grossberg, S., \& Mingolla, E. (1985). Neural dynamics of form perception: Boundary completion, illusory figures, and neon color spreading. Psychological Review, 92, 173-211.

GrossberG, S., \& Mingolla, E. (1987). Neural dynamics of surface perception: Boundary webs, illuminants, and shape-from-shading. Computer Vision, Graphics, \& Image Processing, 37, 116-165.

Kellman, P. , Loukides, M. (1987). An object perception approach to static and kinetic subjective contours. In S. Petry \& G. E. Meyer (Eds.), The perception of illusory contours (pp. 151-164). New York: Springer-Verlag.

Kellman, P. J., \& Shipley, T. F. (1991). A theory of visual interpolation in object perception. Cognitive Psychology, 23, 141-221.

KoFFKA, K. (1935). Principles of Gestalt psychology. New York: Harcourt Brace Co.

Minguzzi, G. F. (1987). Anomalous figures and the tendency to continuation. In S. Petry \& G. E. Meyer (Eds.), The perception of illusory contours (pp. 71-75). New York: Springer-Verlag.

Nakayama, K., Shimojo, S., \& Silverman, G. (1989). Stereoscopic depth: Its relation to image segmentation, grouping, and the recognition of occluded objects. Perception, 18, 55-68.

Petry, S., Harbeck, A., Conway, J., \& Levey, J. (1983). Stimulus determinants of brightness and distinctness of subjective contours. Perception \& Psychophysics, 34, 169-174.

Rock, I., \& ANSON, R. (1979). Illusory contours as the solution to a problem. Perception, 8, 665-687.

Shipley, T. F., \& Kellman, P. J. (1990). The role of discontinuities in the perception of subjective figures. Perception \& Psychophysics, 48, 259-270.

Shipley, T. F., \& Kellman, P. J. (in press). Perception of partly occluded objects and illusory figures: Evidence for an identity hypothesis. Journal of Experimental Psychology: Human Perception \& Performance.

Siegel, S., \& Petry, S. (1991). Evidence for independent processing of subjective contour brightness and sharpness. Perception, 20, 233-241.

Ullman, S. (1976). Filling in the gaps: The shape of the subjective contours and a model for their generation. Biological Cybernetics, $25,1-6$

\section{NOTES}

1. Unit formation refers to the perceptual cohesion of regions of an image. These areas or units are segregated from other units in the image (Koffka, 1935).

2. This generalization may break down at the extremes of viewing distance. At one extreme, the visible parts of an object may become too small to readily detect any separation; at the other extreme, the separation may be so great that the parts of an object are not simultaneously visible.
(Manuscript received May 16, 1991; revision accepted for publication January 27, 1992.) 